\title{
A Safe Start Laparoscopic-assisted Pancreaticoduodenectomy in Songklanagarind Hospital, Thailand: Case Report
}

Tortrakoon Thongkan, M.D., Nan-ak Wiboonkhwan, M.D., Thakerng Pitakteerabundit, M.D., Piyanun Wangkulangkul, M.D., Ph.D.

Department of Surgery, Faculty of Medicine, Prince of Songkla University, Hat Yai, Songkhla 90110, Thailand. Received 8 July 2021 • Revised 7 September 2021 • Accepted 17 September 2021 • Published online 26 October 2021

\section{Abstract:}

Total laparoscopic pancreaticoduodenectomy (PD) is a highly complex procedure. Evidence suggests laparoscopic-assisted PD (LAPD) might be an important link in the process of transition from open PD to total laparoscopic PD. We present the first successful LAPD in a female patient with distal cholangiocarcinoma at Songklanagarind Hospital, Thailand in July 2018. The postoperative course was satisfactory without the clinical relevance of complications. No evidence of recurrence was found after 35 months of follow-up. The results ensured the feasibility and safety of LAPD in the initial phase; regarding both complications and oncological outcomes in a high-volume center for open PD.

Keywords: cholangiocarcinoma, laparoscopic-assisted, pancreaticoduodenectomy, Whipple’s operation

Contact: Nan-ak Wiboonkhwan, M.D.

Department of Surgery, Faculty of Medicine, Prince of Songkla University, Hat Yai, Songkhla 90110, Thailand.

E-mail: boatpsusurgery@gmail.com

2021 JHSMR. Hosting by Prince of Songkla University. All rights reserved.

This is an open access article under the CC BY-NC-ND license

(http://www.jhsmr.org/index.php/jhsmr/about/editorialPolicies\#openAccessPolicy).

J Health Sci Med Res 2022;40(4):481-486 doi: 10.31584/jhsmr.2021849 www.jhsmr.org 


\section{Introduction}

Pancreaticoduodenectomy (PD) operations have been the standard treatment for periampullary cacinoma for decades. However, with the advances in laparoscopic surgery, successful minimally invasive PD (MIPD) has been reported. ${ }^{1}$ Technological and surgical improvements have led to a large number of PD being performed laparoscopically worldwide. However, the complexity of the procedure with the steep learning curve is the limitation in the early experience phase. ${ }^{2}$ Laparoscopic-assisted PD (LAPD) was reported as a linking procedure to total laparoscopic PD with comparable perioperative morbidity, mortality, and oncological outcomes to open PD. ${ }^{3}$ We present the first successful LAPD at Songklanagarind Hospital, tertiary-care center, Thailand, the procedure was performed in July 2018.

\section{Case report}

A 51-year-old female presented with painless progressive jaundice with significant weight loss for 3 months. The computed tomography (CT) scan of the abdomen showed intrahepatic and extrahepatic bile duct dilatation down to the intrapancreatic portion without pancreatic head mass. Blood chemistry results revealed a cholestatic jaundice pattern with $18.60 \mathrm{mg} \%$ of total bilirubin, $15.51 \mathrm{mg} \%$ of direct bilirubin, $532 \mathrm{U} / \mathrm{L}$ of alkaline phosphatase, and 1,438 U/ml of carbonic anhydrase (CA) 19-9. A preoperative endoscopic biliary plastic stent along with a bile duct biopsy was performed. The patient was diagnosed with resectable distal cholangiocarcinoma without distant organ metastasis. Clinical factors such as malnutrition, electrolyte imbalances, and performance status were all optimized before surgery. The operative plan was to perform LAPD for curative aim. Low-molecular-weight heparin $40 \mathrm{mg}$ subcutaneous injection in combination with mechanical intermittent legs compression was used for venous-thromboembolism prophylaxis. The definition and grading of post-pancreatectomy complications were defined according to the International Study Group of Pancreatic Surgery. ${ }^{4}$

Under general anesthesia, the patient was placed with both arms abducted in a supine split-leg posture. The 6-port technique was used; 5 ports comprising working ports, a laparoscopy port, and 1 port at epigastrium for retracting the liver. The location of the ports are shown in Figure 1. The pneumoperitoneal pressure was set at $15 \mathrm{mmHg}$. To obtain good exposure of the surgical field, the gallbladder fundus, and the falciform ligament were retracted in an anterocranial direction using intracorporeal suturing. The LigaSure ${ }^{\circledR}$ (Covidien-Medtronic) Maryland jaw, laparoscopic energy device was utilized for dissection and vascular sealing. The gastrocolic ligament was divided to enter the lesser sac. While the surgeon was on the right side of the patient, extensive kocherization of the duodenum along with the taking down of the hepatic-flexure colon were performed. The middle colic vein was traced back to the superior mesenteric vein (SMV) then a tunnel beneath the neck of the pancreas was created. The pancreatic neck was isolated using umbilical tape. The stomach was divided just proximal to the pylorus using a 60-mm, medium thickness laparoscopic stapler. The lymphadenectomy along the common hepatic artery, hepatic artery proper, main portal vein, and pericholedochal area was carried out while vessels were isolated using vascular loops. The gastroduodenal artery was divided between double hem-o-lok ${ }^{\circledR}$. The common hepatic duct was divided between proximal bulldog clamp and distal silk tie. With the surgeon on the left side of the patient, the ligament of Treitz was split, then proximal jejunum was divided using a laparoscopic stapler. The duodenojejunal segment was then passed underneath the superior mesenteric artery (SMA) to the right side. With the surgeon standing between the legs, the pancreatic neck transection was carried out using a laparoscopic ultrasonic-shear device with care taken not to injure the main pancreatic duct (MPD). The MPD was divided using 


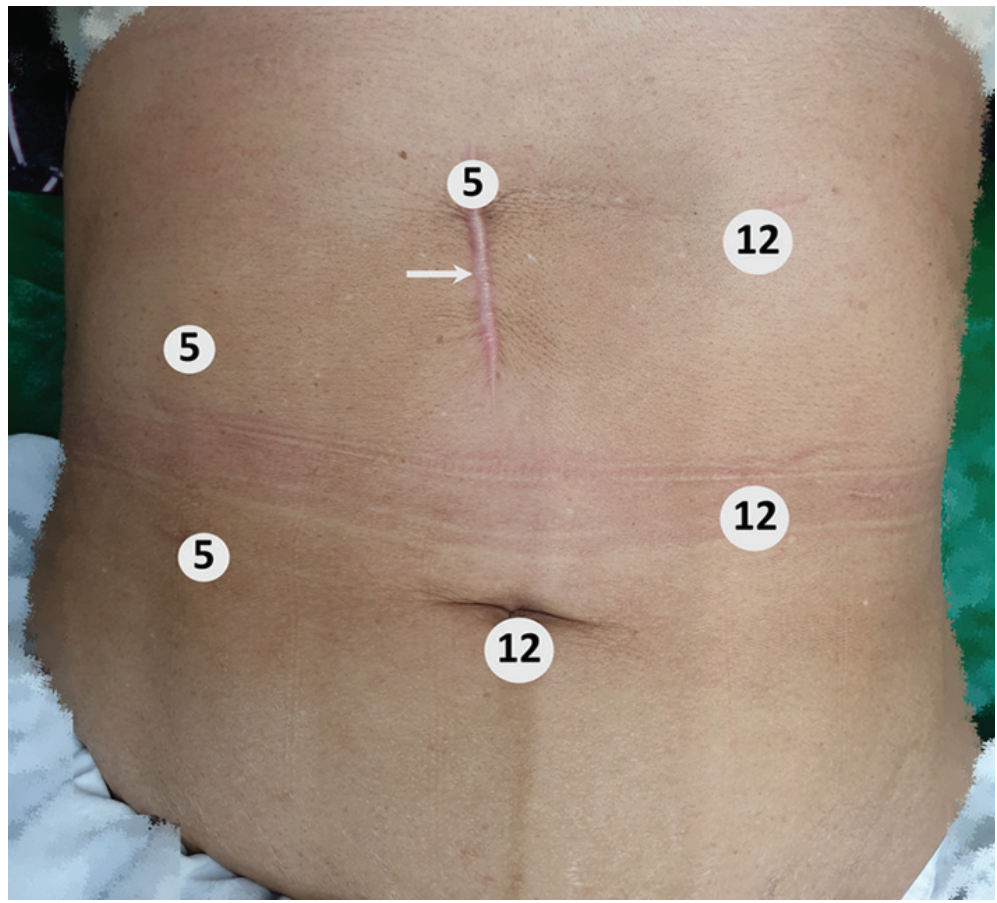

Figure 1 Port position: the 6-port technique was used. Twelve millimeters ports were on the left side of the upper abdomen and one at the infra-umbilicus. These were used as working ports and laparoscopy ports, respectively. The subxiphoid $5 \mathrm{~mm}$ port was used to retract of the liver, with a subsequent, extending incision to $5 \mathrm{~cm}$ mini-exploration (white arrow) for pancreaticojejunostomy and gastrojejunostomy anastomosis. The surgeon stood on the patient's right side, except for when creating the laparoscopic hepaticojejunostomy anastomosis, in which the surgeon stood on the patient's left side. The cameraman stood between the legs.

a laparoscopic scissor and the gastrocolic vein was divided between metallic clips. The pancreatic head and uncinate process were carefully dissected from the right side of the SMV and SMA while the inferior pancreaticoduodenal artery (IPDA) was isolated. The IPDA was divided between double hem-o-lok ${ }^{\circledR}$. Complete cholecystectomy was performed and the specimen was put into an endoscopic bag. Laparoscopic view after complete resection is shown in Figure 2.

The reconstruction phase was performed by hybrid approach; the initial hepaticojejunostomy $(\mathrm{HJ})$ anastomosis was made under laparoscopic view as the surgeon stood on the left side of the patient throughout the biliary reconstruction phase. The retrocolic-jejunal limb was brought through right-sided mesocolon then end-to-side $\mathrm{HJ}$ anastomosis was performed using 4-0 absorbable suture starting from the continuous posterior layer with interrupted anterior layer until complete.

The endoscopic bag was removed through a $5-\mathrm{cm}$ mini-laparotomy at the upper abdomen, this incision was just above the pancreatic stump. A wound protector was then applied. The end-to-side pancreaticojejunostomy (PJ) was performed using 4-0 absorbable suture with dunking technique due to small MPD (1 $\mathrm{mm}$ in diameter) and modified Blumgart's technique for the approximation of seromuscular layer to the pancreatic parenchyma. The endto-side gastrojejunostomy (GJ) anastomosis was carried 


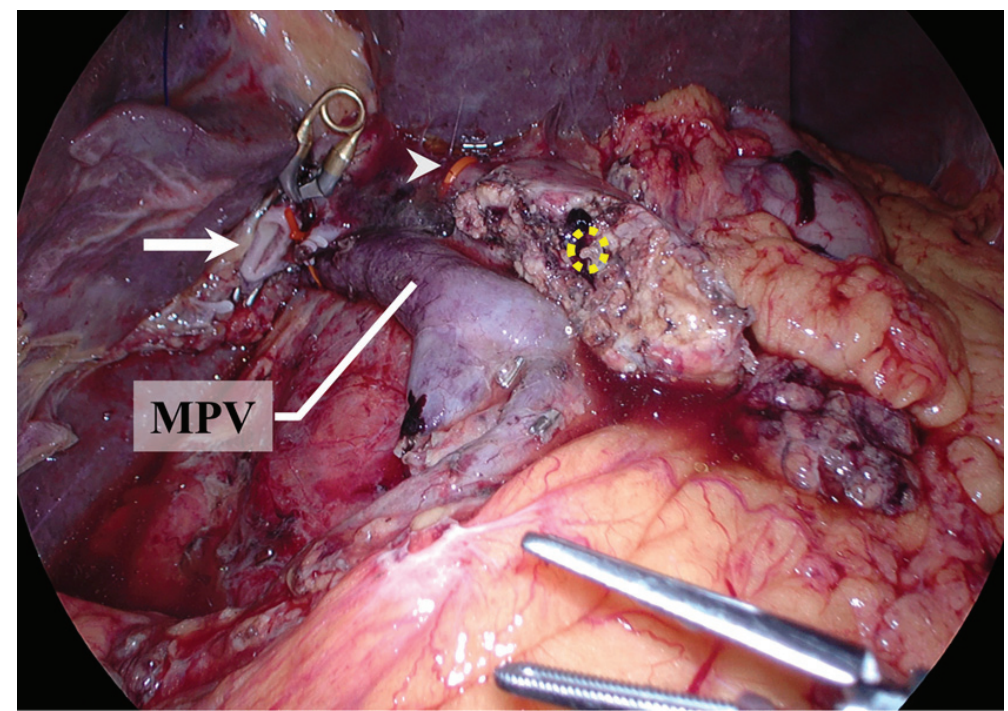

$M P V=$ main portal vein

Figure 2 Laparoscopy view after complete resection: The proximal common hepatic duct was clamped with a bulldog clamp (white arrow). The common hepatic artery (arrowhead) was isolated with vascular looping. The pancreatic stump had a 1-mm diameter of the main pancreatic duct (dot circle) on the cut surface.

out 50-cm away from $\mathrm{HJ}$, the 3-0 absorbable suture with the continuous technique was used for the GJ anastomosis. Finally, a close-suction drain was placed posteriorly to $\mathrm{HJ}$, anteriorly to $\mathrm{PJ}$ anastomosis before the abdominal cavity was closed.

The operative time was 640 minutes and operative blood loss was $500 \mathrm{ml}$ without blood transfusion. There were no intraoperative complications. The pathological result showed adenocarcinoma, well differentiated with invasion to pancreatic head, $4 \mathrm{~cm}$ of tumor size, presence of lymphovascular invasion, perineural invasion, free all surgical margins, negative malignancy of two lymph nodes. There were no clinically relevant postoperative complications. On the first postoperative day (POD), the liquid diet was initiated while the nasogastric tube and the Foley catheter were removed on the second POD. The patient returned to normal ambulation with tolerated liquid and solid food on the third and fourth POD, respectively. The fluid amylase and bilirubin level (on POD 8) from the abdominal drain were 566 U $/$ and $0.59 \mathrm{mg} \%$, respectively, while the drain output was $25 \mathrm{ml} /$ day. The patient was discharged on the eighth POD. The biochemical postoperative pancreatic fistula (POPF) was diagnosed, subsequent drain removal at two weeks was performed without other complications. Postoperative abdominal CT scan, blood chemistry, and CA 19-9 were used for surveillance at 3-month intervals up to 2 years then every 6 months, showing no evidence of disease, the patient was free of jaundice, and cholangitis till the last follow-up at 35 months post-operation.

\section{Discussion}

We present the first successful LAPD in a female patient with distal cholangiocarcinoma. The patient recovered uneventfully, thus ensured the feasibility and 
safety of this complex procedure. Furthermore, the patient remained disease-free after 35-months of follow-up, emphasizing the non-inferiority in terms of the oncological outcome.

Pancreaticoduodenectomy has been the standard treatment of periampullary carcinoma for decades. However, there is insufficient evidence to recommend laparoscopic PD over open PD. ${ }^{5}$ On the other hand, some evidence shows that short-term outcomes and mortality rates were comparable between the two approaches. ${ }^{6}$ Regarding the safety of laparoscopic PD, the high-volume centers of $>10$ total PD/year ${ }^{7}$, and $>20$ total PD/year ${ }^{8}$ were significantly associated with decreased complication, and mortality, respectively. Our center has been routinely performing open PD as the standard treatment of periampullary carcinoma with more than 25 cases/year ${ }^{9}$, thus, this might decrease both morbidity and mortality according to the data mentioned above. In addition, a low morbidity and mortality rate in a high-volume center might reflect the standardized perioperative care in such complex procedures.

Analysis of the learning curve for laparoscopic $\mathrm{PD}$ revealed that operative time, morbidity, and blood loss decreased after 30 to 60 cases. ${ }^{10}$ A large number of cases required during the initial phase reflect the steep learning curve. In this circumstance, LAPD is an alternative procedure before performing total laparoscopic PD. Tian et al. $^{3}$ reported the study of LAPD compared to open PD showed longer operative time in the LAPD group but no difference in clinically relevant POPF, major complication, intraoperative blood transfusion, and $\mathrm{R} 0$ resection rate. The authors suggested that LAPD be used as a bridge between open and total laparoscopic PD. Mentioned above data are the reasons we selected the LAPD as the first MIPD in our center.

Although the first LAPD, the operative time was longer than the mean operative time of open PD in our center (640.0 and 485.1 mins, respectively) ${ }^{9}$, the operative blood loss seems no different. In addition, the postoperative hospital stay was shorter than the previously reported LAPD series by Cho et al. ${ }^{11}$ and similar to open PD in our center. The reported incidence of biochemical POPF was $40.0 \%{ }^{12}$, and the clinically relevant POPF (grade $\mathrm{B} / \mathrm{C}$ ) ranges from $8.8 \%$ to $12.0 \%{ }^{13}$. Even though small MPD less than $5 \mathrm{~mm}$ is the significant risk factor for developing POPF $^{14}$, our patient had no clinically relevant POPF with only $1 \mathrm{~mm}$ MPD diameter. The mini-laparotomy over the pancreatic stump may attribute to precise anastomosis mitigating the development of severe POPF.

The oncological outcomes of laparoscopic PD are similar to open $\mathrm{PD}^{7}$. Moreover, in one report the progression-free survival was longer in minimally invasive group. Gilbaud et al. ${ }^{15}$ reported 3-year disease-free survival of $13.5 \%$, with tumor size more than $2 \mathrm{~cm}$ was significant associated with worse outcome in multivariate analysis. Although the presence of many worse predictive factors associated with recurrence disease in our cases such as inadequate lymph node examination, large tumor size more than $2 \mathrm{~cm}^{15}$, lymphovascular invasion, perineural invasion, the patient remained disease-free after 35 months of follow-up. Therefore, an acceptable oncological outcome was demonstrated in this case.

\section{Conclusion}

Although the PD procedure is a high complexity procedure, we present the first successful LAPD in a female patient with distal cholangiocarcinoma. The results ensured the feasibility and safety in terms of both complication and oncological outcomes in a high-volume center for PD. Hence the need for further prospective evaluation of outcomes in MIPD.

\section{Conflict of interest}

None declared 


\section{Acknowledgement}

We thank Prisuda Bualoy and Kiraporn Ruangnuy, currently chief of the operating room at Songklanagarind Hospital, Prince of Songkla University, Thailand, for providing the great laparoscopic setup and also taking care of our patient.

\section{References}

1. Gagner M, Pomp A. Laparoscopic pylorus-preserving pancreatoduodenectomy. Surg Endosc 1994;8:408-10.

2. Kuroki T, Kitasato A, Adachi T, Tanaka T, Hirabaru M, Matsushima $\mathrm{H}$, et al. Learning curve for laparoscopic pancreaticoduodenectomy: a single surgeon's experience with consecutive patients. Hepatogastroenterology 2014;61:838-41.

3. Tian F, Wang YZ, Hua SR, Liu QF, Guo JC. Laparoscopic assisted pancreaticoduodenectomy: an important link in the process of transition from open to total laparoscopic pancreaticoduodenectomy. BMC Surg 2020;20:89.

4. Bassi C, Marchegiani G, Dervenis C, Sarr M, Abu Hilal M, Adham M, et al. The 2016 update of the International Study Group (ISGPS) definition and grading of postoperative pancreatic fistula: 11 Years After. Surgery 2017;161:584-91.

5. Asbun HJ, Moekotte AL, Vissers FL, Kunzler F, Cipriani F, Alseidi A, et al. The Miami International Evidence-based Guidelines on Minimally Invasive Pancreas Resection. Ann Surg 2020;271: $1-14$.

6. van Hilst J, de Rooij T, Bosscha K, Brinkman DJ, van Dieren $\mathrm{S}$, Dijkgraaf MG, et al. Laparoscopic versus open pancreatoduodenectomy for pancreatic or periampullary tumours (LEOPARD-2): a multicentre, patient-blinded, randomised controlled phase 2/3 trial. Lancet Gastroenterol Hepatol 2019; 4:199-207.

7. Torphy RJ, Friedman C, Halpern A, Chapman BC, Ahrendt SS, McCarter MM, et al. Comparing short-term and oncologic outcomes of minimally invasive versus open pancreaticoduodenectomy across low and high volume centers. Ann Surg 2019;270:1147-55.

8. Tran TB, Dua MM, Worhunsky DJ, Poultsides GA, Norton JA, Visser BC. The first decade of laparoscopic pancreaticoduodenectomy in the United States: costs and outcomes using the nationwide inpatient sample. Surg Endosc 2016;30:177883.

9. Thongkan T, Pitakteerabundit T, Wiboonkhwan N. Does pyloricring resection in pancreaticoduodenectomy prevent delayed gastric emptying? J Health Sci Med Res 2021;39:303-12.

10. Lu C, Jin W, Mou YP, Zhou J, Xu X, Xia T, et al. Analysis of learning curve for laparoscopic pancreaticoduodenectomy. J Vis Surg 2016;2:145.

11. Cho A, Yamamoto $H$, Nagata M, Takiguchi $N$, Shimada $H$, Kainuma $\mathrm{O}$, et al. Comparison of laparoscopy-assisted and open pylorus-preserving pancreaticoduodenectomy for periampullary disease. Am J Surg 2009;198:445-9.

12. Tan JKH, Ng JJ, Yeo M, Koh FHX, Bonney GK, Ganpathi IS, et al. Propensity score-matched analysis of early outcomes after laparoscopic-assisted versus open pancreaticoduodenectomy. ANZ J Surg 2019;89:190-4.

13. Chen K, Pan Y, Huang CJ, Chen QL, Zhang RC, Zhang MZ, et al. Laparoscopic versus open pancreatic resection for ductal adenocarcinoma: separate propensity score matching analyses of distal pancreatectomy and pancreaticoduodenectomy. BMC cancer 2021;21:382.

14. Akgul O, Merath K, Mehta R, Hyer JM, Chakedis J, Wiemann B, et al. Postoperative pancreatic fistula following pancreaticoduodenectomy-stratification of patient risk. J Gastrointest Surg 2019;23:1817-24.

15. Guilbaud T, Girard E, Lemoine C, Schlienger G, Alao O, Risse $\mathrm{O}$, et al. Intra-pancreatic distal cholangiocarcinoma and pancreatic ductal adenocarcinoma: a common short and longterm prognosis? Updates Surg 2021;73:439-50. 